\title{
Cholesterol Modulates Huntingtin Binding to and Aggregation on Lipid Membranes
}

Xiang Gao

Follow this and additional works at: https://researchrepository.wvu.edu/etd

\section{Recommended Citation}

Gao, Xiang, "Cholesterol Modulates Huntingtin Binding to and Aggregation on Lipid Membranes" (2015). Graduate Theses, Dissertations, and Problem Reports. 5646.

https://researchrepository.wvu.edu/etd/5646

This Thesis is protected by copyright and/or related rights. It has been brought to you by the The Research Repository @ WVU with permission from the rights-holder(s). You are free to use this Thesis in any way that is permitted by the copyright and related rights legislation that applies to your use. For other uses you must obtain permission from the rights-holder(s) directly, unless additional rights are indicated by a Creative Commons license in the record and/ or on the work itself. This Thesis has been accepted for inclusion in WVU Graduate Theses, Dissertations, and Problem Reports collection by an authorized administrator of The Research Repository @ WVU. For more information, please contact researchrepository@mail.wvu.edu. 


\title{
Cholesterol Modulates Huntingtin Binding to and Aggregation on Lipid Membranes
}

\author{
Xiang Gao \\ Thesis submitted \\ to the Eberly College of Arts and Sciences \\ at West Virginia University
}

in partial fulfillment of the requirements for the degree of

Master of Science in

Chemistry/Physical Chemistry

Justin Legleiter, Ph.D., Chair

Blake Mertz, Ph.D.

Stephen Valentine, Ph.D.

Department of Chemistry

Morgantown, West Virginia

2015

Keywords: Huntington disease, huntingtin, amyloid, cholesterol, lipid bilayers, protein aggregation, polyglutamine, AFM, PDA, morphology

Copyright 2015 Xiang Gao 


\title{
ABSTRACT \\ Cholesterol Modulates Huntingtin Binding to and Aggregation on Lipid Membranes
}

\begin{abstract}
Xiang Gao
Huntington disease is an autosomal dominant neurodegenerative disorder. The abnormally long CAG-repeats in the huntingtin gene that encode an expanded polyglutamine stretch which promotes self-assemble of huntingtin into different aggregation species that are disease related. Huntingtin intimately interacts with a variety of lipid membranes. Lipid composition is altered in HD, especially cholesterol content. Here we investigate how cholesterol content modulates the interaction between huntingtin and lipid membranes. TBLE/PDA binding assay is performed to test the binding affinity of huntingtin with lipid bilayers containing different amount of cholesterol. As the cholesterol content increases, the extent of huntingtin binding to lipid bilayers decreases. Also, atomic force microscopy (AFM) is used to directly monitor the formation of aggregates on supported lipid bilayers containing exogenously added cholesterol. Morphological and mechanical changes in the bilayers exposed to huntingtin are observed by the presence of cholesterol. On pure TBLE, globular aggregates are formed and grainy in appearance. Most of the bilayers are disrupted. In contrast, lipid bilayers enriched in different amount of cholesterol facilitate the formation of plateau-like aggregates with a smooth appearance. With the increase of cholesterol content in lipid bilayers, the percentage of the surface disrupted by the protein aggregation decreases. In sum, the presence and amount of cholesterol in lipid bilayers modulates the huntingtin binding and aggregation on lipid membranes.
\end{abstract}




\section{Table of Contents}

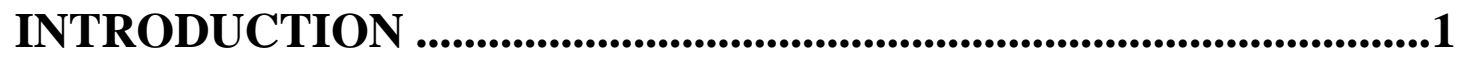

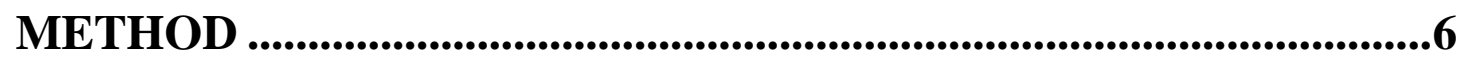

EXPERIMENT PROCEDURES.......................................................99

RESULTS

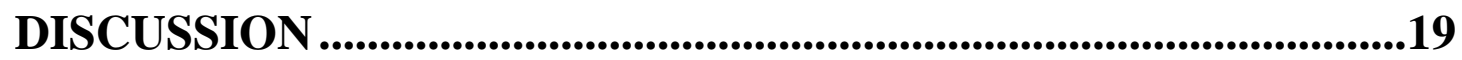

CONCLUSION

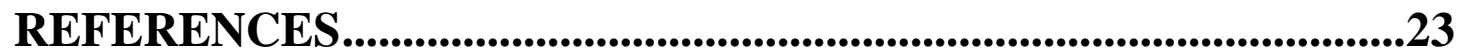

Acknowledgement ............................................................................................30 


\section{INTRODUCTION}

A common feature of neurodegenerative diseases such as Alzheimer's disease (AD), Parkinson's disease (PD), and Huntington's disease (HD), is the formation of protein aggregates that deposit in tissues or cellular compartments [1]. Non-native conformation of specific proteins promotes their self-assembly into insoluble aggregates. These diseases related aggregates are comprised of extend, $\beta$-sheet rich fibrils, referred to as amyloid, and thus these diseases are referred to as amyloid disease. Amyloid formation typically occurs via the nucleation-growth mechanism, which is comprised of a lag phase and a growth phase [1-3]. However, the assembly process of aggregates can adopt a myriad of complex aggregation pathways (Figure 1). A variety of aggregate species (e.g. oligomers, amorphous aggregates, protofibrils, and fibrils) can be observed along the aggregation pathway.

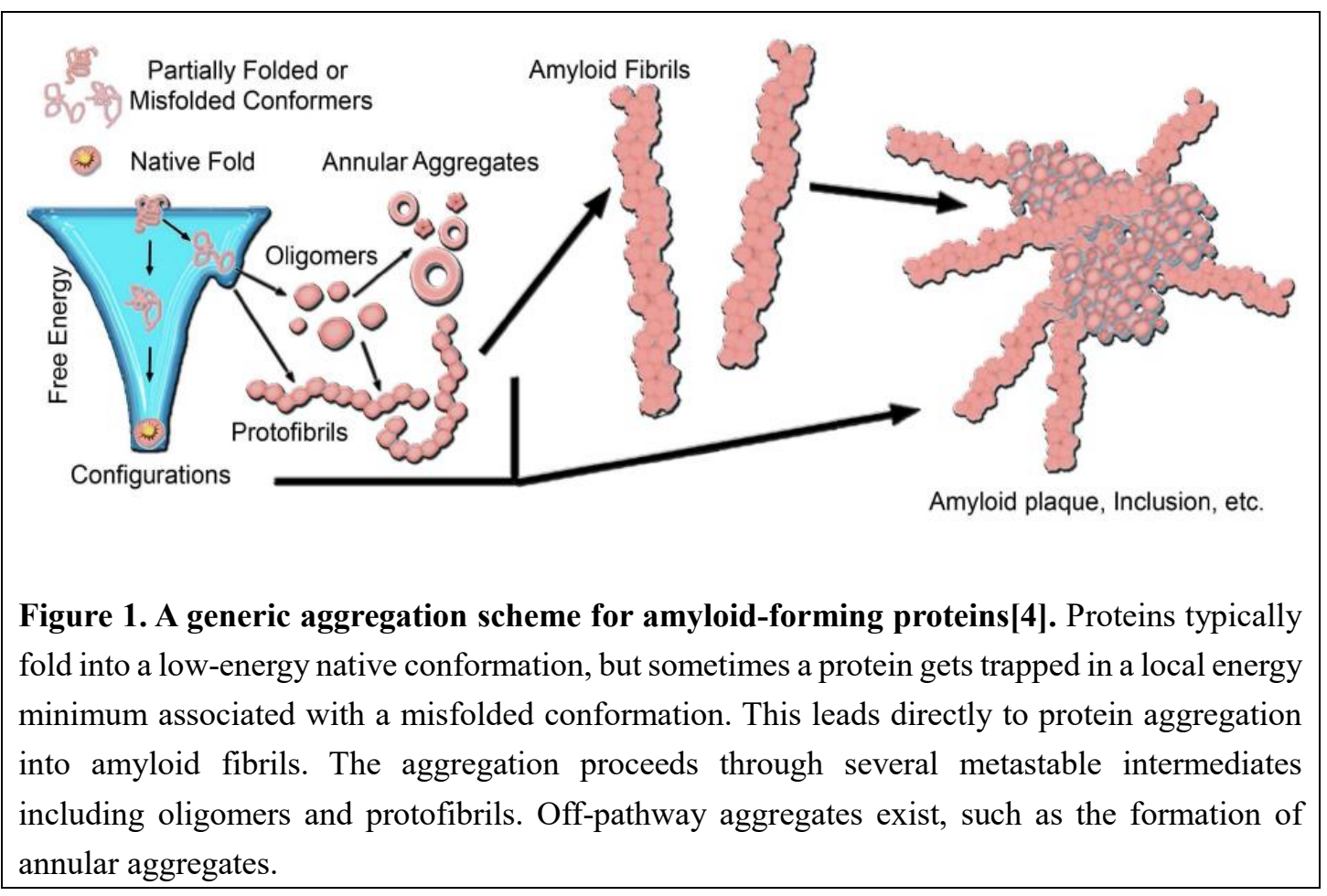


Environment can influence the formation of protein aggregates, and one such environmental factor is the presence of surfaces. Conformational changes can be induced when proteins encounter surfaces [5]. Different aggregate morphologies can be promoted by different solid surfaces. A classic example of this is the aggregation of $\mathrm{A} \beta$, a protein associated with Alzheimer's disease, aggregating on mica [6] or graphite [7]. While $\mathrm{A} \beta$ forms oligomers that coalesce into protofibrils with a beaded morphology on mica, it forms extended, flat nano-ribbons on graphite that are epitaxially oriented. Like solid surfaces, lipid membrane can also alter the aggregation process. For most amyloid proteins, the formation of fibrils is accelerated in the presence of lipid membranes compared to bulk solution [8-10]. Amyloid-forming proteins can bind and/or insert into lipid membrane and accumulate within cellular or subcellular membranes $[11,12]$, accelerating the nucleation process. Lipid composition within a membrane can also influence the aggregation process [13].

$\mathrm{HD}$ is a progressive neurological disorder. Psychiatric manifestations, cognitive loss, and chorea [14] are the typical symptoms. Neuronal cell loss occurs mainly in the cerebral cortex and striatum with up to $30 \%$ of brain mass [15]. HD is inherited by a dominant genetic mutation in an autosomal fashion. The genetic mutation is abnormally long CAG-repeats in the huntingtin gene which encodes an extended polyglutamine (polyQ) stretch near the N-terminus of the huntingtin (htt) protein. In brain tissues, polyQ directly causes htt protein to aggregate into different types of aggregates, eventually forming cytoplasmic and nuclear inclusion bodies [16] which are the hallmarks of HD. Inclusion bodies are commonly thought to be the pathological agents 
by potentially sequestering or incorporating some important proteins [17]. The age of onset and disease severity are tightly correlated with the length of polyQ with a threshold $\sim 35$ glutamines required for disease [18]. The presence of expanded polyQ promote the formation of fibrillary structures rich in $\beta$-sheet content (amyloid fibril). But the assembly of mutant htt into mature amyloid fibril can adopt various aggregation pathways. There are several metastable intermediate aggregate species along the pathway such as oligomers and amorphous aggregates, which are associated with varying degrees of toxicity [19]. It has been suggested that the small diffusible aggregates like oligomer are more toxic and that the mature fibril are inert or potentially protective [20]. The most toxic pathologic species remain controversial.

Htt aggregation adopts the nucleation-growth mechanisms. The nucleation event is critical to the initiation of aggregation; although, the event is a slow process and thermodynamically unfavorable. Once nucleated, aggregation proceeds by incorporating htt monomers into different aggregate species leading to the growth phase. For pure polyQ peptides, the nucleus is thought to be a monomer which undergoes a conformational change [21]. Once flanking sequences associated with htt are added to polyQ peptides, a critical tetramer nucleus has been observed [22, 23].

Full length htt is more than 3000 amino acids long and it can be cleaved by several proteases. Although there are many htt $\mathrm{N}$-terminal fragments, the exon 1 has a potentially important role in HD. Exon1 fragment can be detected in transgenic mice expressing full length htt [24] and in HD patients [25] due to the aberrant cleavage of full length htt in polyQ length dependent manner [26]. In knock-in mouse model, 
expression of exon1 with an expanded polyQ leads to a progressive neurological phenotype $[16,27]$.

$\mathrm{Htt}$ is a multifunctional protein and is present in mammals. Expression of htt is essential for brain development and neuronal survival [28]. In mouse model, knockout of htt gene is lethal [29]. Htt plays an important role in vesicle trafficking i.e. the transport of lipid vesicles (endocytic, synaptic or lysosomal), especially along microtubules [30-33]. In membrane fractions from brains, htt is detected [34]. Htt can localize to various subcellular compartments as its normal function [35]. Specifically, htt binds to ER reversibly and to mitochondria [36, 37]. Htt inclusion bodies incorporate multi-vesicular membrane into their surfaces [38, 39]. All of this implicates that htt intimately interacts with various lipid membranes. Although full length htt has multiple lipid binding sites [40], the highly conserved first 17 amino acid $\left(\mathrm{Nt}^{17}\right)$ in the $\mathrm{N}$ terminus is thought to target lipid membranes. In the presence of lipid membranes, $\mathrm{Nt}^{17}$ has an induced $\alpha$ helix structure [41]. Furthermore the flank polyQ domain promotes the insertion of htt into lipid membrane [42]. Htt disrupts lipid bilayers in a polyQ length dependent manner [43].

Cholesterol is a main component of biological lipid membranes, especially in neurons. Approximately $25 \%$ of the lipids content in neuronal membranes is cholesterol, and $70 \%$ of myelin sheaths is comprised of cholesterol [44]. All of this indicates that cholesterol plays an important role in brain tissues. Structurally, cholesterol influences membrane's thickness and fluidity [45]. Functionally, the important property of electrical insulation for neuron cells is related to the cholesterol's ability to limit ion 
leakage through membrane [46]. Also, cholesterol affect neurotrophic factors, transmitter receptors and signal reduction that are specific to neuron cells [47]. Cholesterol does not cross the blood brain barrier, so all the cholesterol in the central nervous system is synthesized endogenously which is a complex process and can be affected by many factors.

Altered cholesterol homeostasis is observed in cellular and animal models of HD, as well as in HD patient tissues [48-50]. Although cholesterol biosynthesis is reduced in $\mathrm{HD}$, the specifics of altered cholesterol content remains controversial. A decrease in cholesterol content is reported [51-54] by numerous studies. An increase in cholesterol levels are also demonstrated by other reports [55-58]. Many of these discrepancies may be associated with methodology and model systems used to study cholesterol levels in HD.

The role of htt aggregation in HD is well established; however, investigation of environmental factors which can influence the aggregation process is still important. Although the presence of lipid membrane can change htt aggregation, the role of lipid composition in modulating htt aggregation is poorly understood. The fact that cholesterol is important to neurons and the hemostasis of cholesterol in HD is altered indicates that cholesterol may play an important role in HD. Here, we characterize the interaction of htt exon1 fragments with total brain lipid extract (TBLE) lipid membranes containing different amount of exogenously added cholesterol. 


\section{METHODS}

TBLE/PDA lipid vesicle protein binding assay: Conjugated polydiacetylene (PDA) is polymer which has a long conjugation network and exhibits chromatic properties. Experiencing surface pressure resulting in the perturbation of the PDA dependent side chains, PDA can have a rapid irreversible blue-red colorimetric transitions due to a subtle induced structural transition that shortens the effective conjugation network of the backbone of the polymer. Hydrophilic head group and hydrophobic tail that

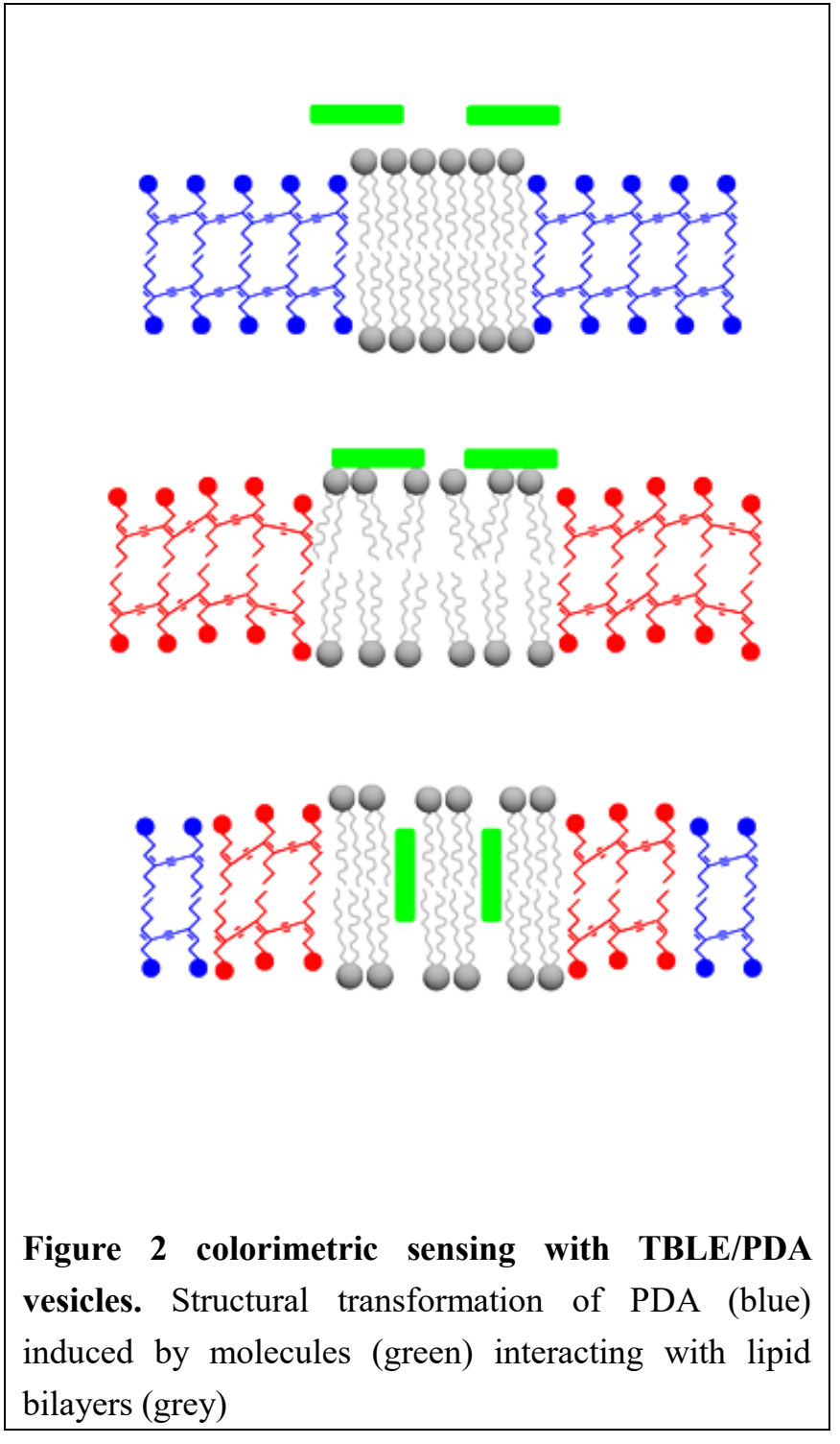

Figure 2 colorimetric sensing with TBLE/PDA vesicles. Structural transformation of PDA (blue) induced by molecules (green) interacting with hipid bers (grey)

give the PDA lipidomimetic structure promotes lipid/PDA mixing and formation of biomimetic membrane assemblies. It has been shown that biological analytes primarily interacting with the lipid domains can still induce the blue-red transformations. Although the mechanism has not been fully elucidated, it's been proposed that the interaction between analytes and lipid domains can influence the structure of PDA through the lipid/polymer interface. Compared to the moderate blue-red transformation generated by the deeper penetration into the hydrophobic core, the surface disturbance 
interacting with the lipid head group can induce more pronounce color transition, i.e. protein/lipid binding [59](Figure 2). Through the blue-red colorimetric change of the PDA/lipid vesicles aggregation of amyloid proteins to membrane can be studied [6063]. Furthermore, this transition can be quantified and obtained by determining the percentage colorimetric response (\%CR), defined as [60, 62]

$$
\% \mathrm{CR}=\left[\left(\mathrm{PB}_{0}-\mathrm{PB}_{\mathrm{I}}\right) / \mathrm{PB}_{0}\right] \times 100
$$

where $\mathrm{PB}$ is a red/blue ratio of absorbance $(\mathrm{A})$ defined as $\mathrm{A}_{\text {blue }} /\left(\mathrm{A}_{\text {blue }}+\mathrm{A}_{\text {red }}\right)$. $\mathrm{A}_{\text {blue }}$ is the "blue" component in the UV-vis spectrum $(\approx 640 \mathrm{~nm}) ; \mathrm{A}_{\text {red }}$ is the "red" component $(\approx 500 \mathrm{~nm}), \mathrm{PB}_{0}$ is the red/blue ratio of the control sample (before induction of color change), and $\mathrm{PB}_{\mathrm{I}}$ is the value obtained for the vesicle solution after addition of peptides.

Atomic force microscopy: AFM is an important technique for imaging with nanoscale spatial resolution. Unlike the optical microscopy suffering from the spacial resolution limitations mainly due to the diffraction limit of light, a cantilever is used to interact directly with the sample to collect the sample surface information that is used to reconstruct the sample surface topography. In AFM, the cantilever is allowed to physically interact with the sample. A laser beam is projected into the sharp tip of the cantilever and reflected onto a photo detector to generate an optical lever. Through the optical lever, the vertical movement of the cantilever is amplified and monitored. In tapping mode AFM, the cantilever is harmonically driven near the resonance frequency. 
When the cantilever interacts with the sample by intermittently making contact at the bottom of an oscillation cycle, the oscillation amplitude is changed to a tapping amplitude. By maintaining the tapping amplitude to a pre-determined value, or set-point, while raster scanning the probe across the surface, the sample topography and other properties can be characterized. Both height image and phase image can be obtained. In phase imaging, the shift in the phase of the oscillating probe tip with respect to the oscillating drive is mapped and provide information about the compositional contrast of the surface. In solution, local elasticity of lipid membrane can be mapped because of the sensitivity of phase-shifts to local elastic response [64]. Topographic information is indicated by the height image while phase image is sensitive to variations in composition, adhesion, friction, viscoelasticity as well as other factors [64]. In recent years, AFM has become a particularly useful technique in investigating disease related protein aggregation process, especially amyloid formation. Furthermore, tapping mode AFM can work in solution, providing the opportunity to monitor protein aggregation under physiologically-relevant conditions. From small oligomers to huge mature fibrils, aggregation species can be observed on surfaces such as lipid membranes $[6,7,9,10$, $43,65,66]$. Continuously imaging the same surface area allows for tracking the fate of individual aggregates and changes in morphology. 


\section{EXPERIMENT PROCEDURES}

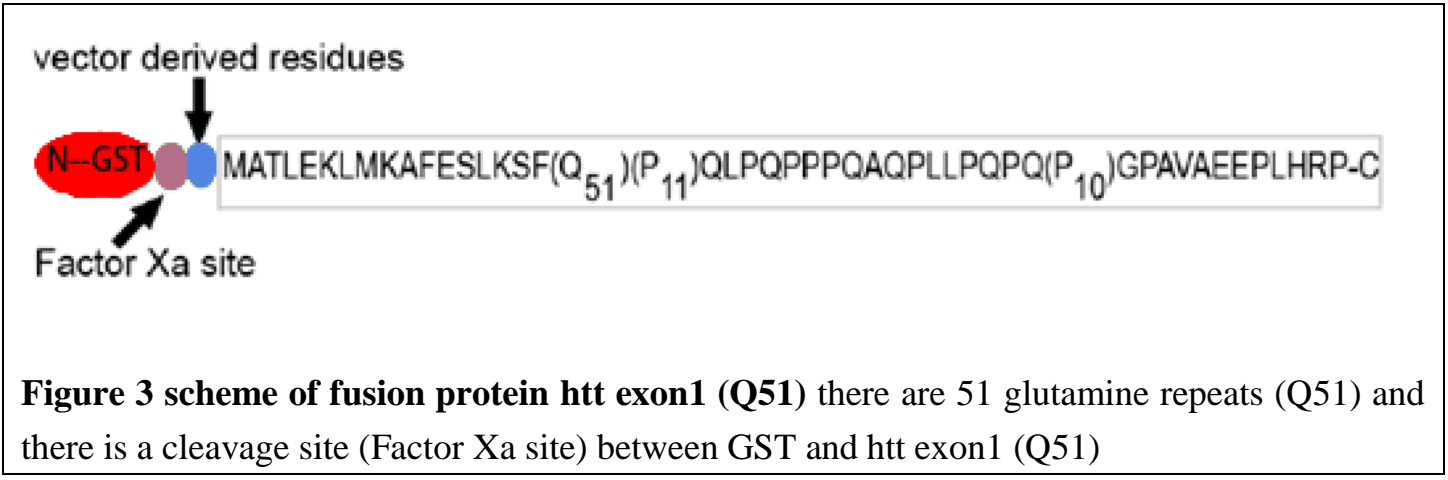

Purification of GST-htt-exon1 fusion proteins: Expression of GST-htt fusion proteins was accomplished by transfecting Escherichia coli with the appropriately designed plasmid (Figure 3). After growing an appropriate concentration of E.coli in LB broth, isopropyl $\beta$-D-thiogalactoside was added to induce the expression of the fusion protein at $30^{\circ} \mathrm{C}$ for $4 \mathrm{~h}$. The cells were pelleted by centrifugation. Protease inhibitor was added, and the cells were lysed by adding $0.5 \mathrm{mg} / \mathrm{mL}$ lysosome. Ultrasonication was used to further break the cells and release fusion protein. A GST affinity column was used to purify the lysate by liquid chromatography. Relevant fractions were collected, and SDS-PAGE electrophoresis was performed to verify the product and determine purity. Protein was concentrated by centrifugal filter after 48 hours of dialysis. Pre-existing aggregates were removed by high spin centrifuge at 20,000g for 30min. Cleavage of the GST moiety was achieved by Factor Xa. Cleavage initiates aggregation. Prior to addition to any lipid membranes, fusion protein and Factor Xa were incubated on ice for 1 hour to generate enough cleaved htt protein for the assay. TBLE/PDA lipid vesicle protein binding assay: TBLE (Avanti Polar Lipids), diacetylene monomers 10,12-tricosadiynoic acid (GFS Chemicals, Columbus, OH) and 
an appropriate amount of cholesterol was dissolved in a solution of 1:1 chloroform/ethanol. The molar ratio of total lipid (TBLE + cholesterol) to PDA was 2:3. Rotary evaporator was used to evaporate the solution, leaving a thin, dry film on the round bottled flask. $70{ }^{\circ} \mathrm{C}$ Tris-buffered saline (TBS) was added to the flask and sonicated at $100 \mathrm{~W}$ using a sonic dismembrator (FisherSci) to resuspend the mixture and generate TBLE/PDA vesicles. The suspension was stored in a beaker at $4{ }^{\circ} \mathrm{C}$ overnight to ensure self-assembly of vesicles. The diacetylene monomers were polymerized by irradiation at $254 \mathrm{~nm}$ with 7 lumens for 10 minutes with stirring, resulting in a brilliant blue color. Htt-exon1(Q51) and polymerized TBLE/PDA was added into the wells of 96 well plate. As controls, TBS (negative control) and $\mathrm{NaOH}$ ( $\mathrm{pH} 12)$ (positive control) were added into the wells containing htt-exon1(Q51). The final concentration of htt-exon1(Q51) was $20 \mu \mathrm{M}$ in each well. Each experiments were performed in triplicate in a 96-well format. The colorimetric response of each well was recorded over 16h using an Infinite M1000 Pro plate reader (TECAN, Switzerland) at room temperature measuring both the blue component $(640 \mathrm{~nm})$ and the red component $(500 \mathrm{~nm})$ of the spectrum.

Preparation of supported TBLE bilayers for AFM experiments: TBLE (Avanti Polar Lipids) and cholesterol (Avanti Polar Lipids) were separately dissolved in chloroform (Fisher Scientific). Based on mass, 10\%, 20\% or 30\% exogenous cholesterol was added to TBLE. The chloroform was then evaporated off to form a lipid film. These films were resuspended in PBS at a concentration of $0.5 \mathrm{mg} / \mathrm{ml}$ by vortexing and sonication. A series of 5 freeze-thaw cycles promoted the formation of bilayers and multilayer lipid 
sheets. Bath sonication was then performed for 8 minutes to promote the formation of lipid vesicles from the lipid sheets. $35 \mu \mathrm{L}$ of the vesicle solution was injected into the AFM fluid cell and exposed to freshly cleaved mica. By fast continual AFM scanning, the cantilever aided in fusing the lipid vesicles into a supported lipid bilayer. $35 \mu \mathrm{L}$ of PBS was then injected into the fluid cell and sucked out three times to remove excess lipid vesicles.

AFM imaging condition: In situ AFM experiments were performed with a Nanoscope V MultiMode scanning probe microscope (Bruker, Santa Barbara, CA) equipped with a sealable fluid cell and a closed-loop "vertical engage" J-scanner. Silicon cantilevers (VISTA probes) with a nominal spring constant of $0.1 \mathrm{~N} / \mathrm{m}$ were used to capture images. Scan rates were set to $\sim 1.95 \mathrm{~Hz}$ with cantilever drive frequencies ranging from $\sim 4$ to 8 $\mathrm{kHz}$. The tapping amplitude was set at about $75 \%$ of free amplitude which was $\sim 25 \mathrm{~nm}$. AFM images were processed with custom written scripts that use the Matlab image processing toolbox (Mathworks, Natick, MA). 


\section{RESULTS}

Enriching TBLE with cholesterol

alters the extent of htt-exon1

binding to lipid vesicles as

determined by a TBLE/PDA

binding assay: To verify that

altering the lipid content of TBLE

would modulate the interaction of

full-length htt-exon1 with lipids,

we performed a TBLE/PDA

vesicle lipid binding assay (Figure

4). Four experiments were

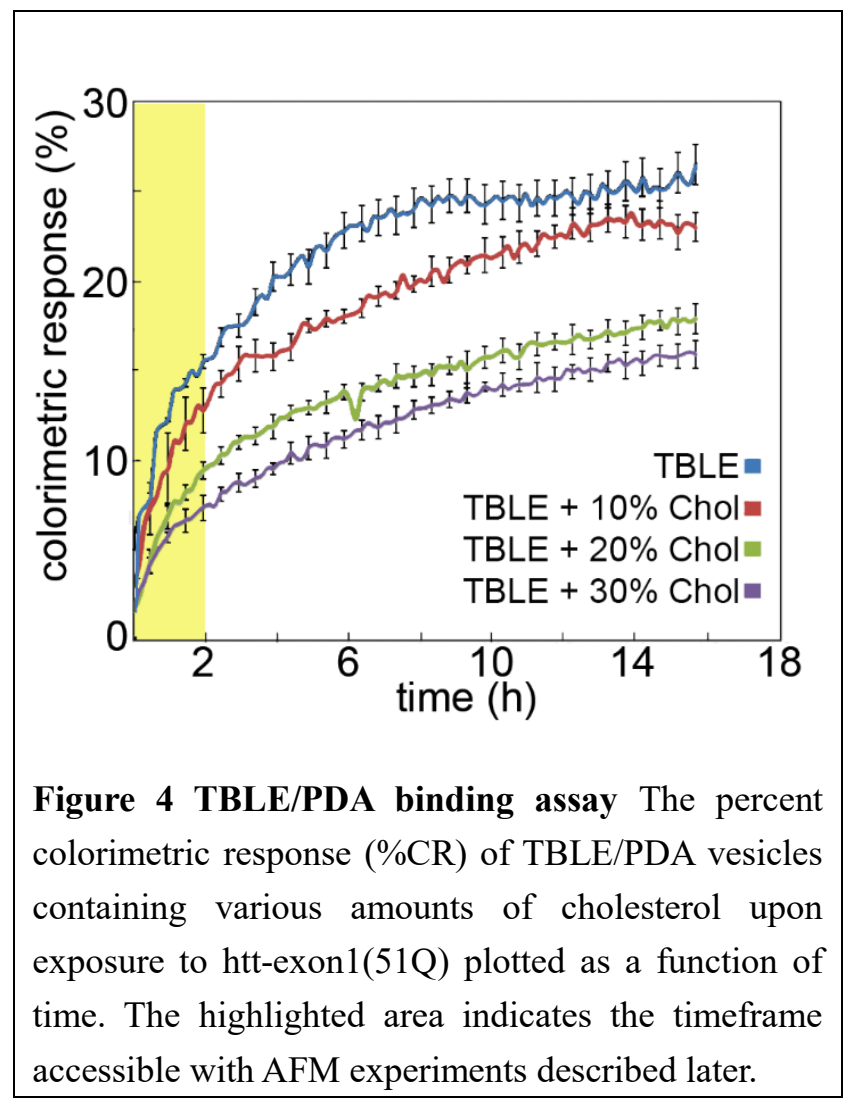

performed with TBLE/PDA vesicles enriched with 0\% (pure TBLE), 10\%, 20\%, 30\%

exogenous cholesterol by mass. The vesicles were exposed to $20 \mu \mathrm{M}$ htt-exon1 (Q51),

and the colorimetric response was continuously measured for 16 hours. For every experiment, once the vesicles are exposed to htt-exon1 (Q51) an initial steady increase in \% CR starts. With time, the \%CR finally levels off to a stationary state. With increasing amounts of cholesterol, the initial increase in \%CR was less steep, indicating the binding of htt-exon1 (Q51) to the vesicles was inhibited. Also, as the exogenous cholesterol content was enriched from $0 \%$ to $30 \%$, the final stationary state $\% \mathrm{CR}$ decreased, which indicates that the overall protein lipid binding was reduced. Still, there was significant interaction between htt-exon1 (Q51) and lipid vesicles even for 
TBLE/PDA vesicles containing 30\% exogenous cholesterol.

Cholesterol modulates the morphological and mechanical changes in lipid bilayers associated with exposure to htt-exonl (Q51): To investigate the influence of cholesterol on the aggregation of htt on lipid membranes, supported TBLE bilayers containing various amounts of cholesterol were exposed to htt-exon1 (51Q) and continuously imaged using in situ AFM, allowing for the direct tracking of protein aggregation on the bilayer. Supported lipid bilayer can be formed by fusion of lipid vesicles on the hydrophilic surface of mica. There are various kinds of forces between the lipid bilayer and mica which stabilized the bilayer near the surface. However, there is still a gap between the mica and the bottom leaflet of the bilayer [67]. The supported lipid bilayer still retains many free membrane properties i.e. lateral fluidity [67]. As a result, supported lipid bilayers are an appropriate model surfaces to study the interaction of amyloid-forming proteins with lipid membranes [9, 10, 43, 65, 66]. Height images (surface topography) and phase images were obtained simultaneously.

For every experiments, only a small size lipid bilayer $(10 \times 10 \mu \mathrm{m})$ was continuously monitored by AFM. However, a large area (40 x $40 \mu \mathrm{m}$ in size) surrounding this small patch of lipid was verified before exposure to htt-exon1 (51Q). The unperturbed bilayers typically had a RMS roughness value less than $0.2 \mathrm{~nm}$. First, it was confirmed that stable, supported TBLE enriched with different amounts of exogenous cholesterol could form smooth, structurally stable bilayers. Bilayers indeed formed, and there was no indication of distinct lipid domains or structural instability in either AFM height or phase images for several hours (Figure 5). 
While only a $10 \times 10 \mu \mathrm{m}$ patch was continuously monitored in the AFM experiments, the scan area was intermittently off-set to image an area of the larger bilayer that had not been previously imaged. This was done as a control to make sure that the scanning process was not influencing the aggregation process.

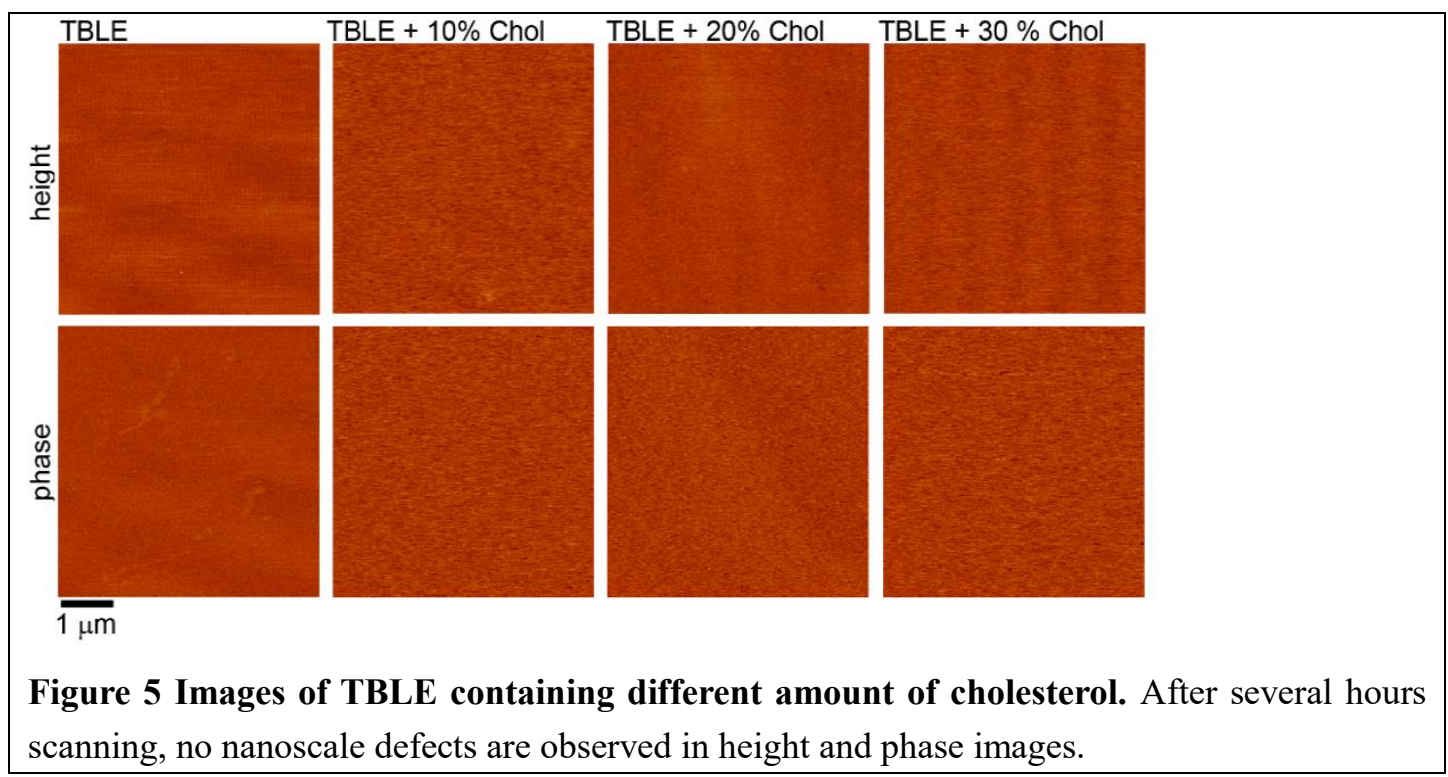

For all the experiments, the supported lipid bilayers with varying amounts of added cholesterol were exposed to freshly prepared aliquots of htt-exon1 (Q51) at a final protein concentration of $20 \mu \mathrm{M}$ in the fluid cell. When pure TBLE was exposed to 20 $\mu \mathrm{M}$ solutions of htt-exon1 (Q51), large areas of the bilayer were disrupted extensively and immediately; although, some small regions appeared unaffected (Figure 6). In the disrupted regions, RMS surface roughness increased, and discrete aggregates accumulated. With time, the disrupted areas expanded and became even rougher. The aggregates also increased in number and size.

In the AFM phase images, there was distinct contrast between the affected area and unperturbed regions, and this contrast remained consistent during the 120minutes observation. This suggests that there are mechanical differences between 
these different regions of the bilayer. This observation is consistent with previous reports that similar morphological changes in bilayers exposed to htt were associated a decreased compression modulus of the membrane $[65,68]$.

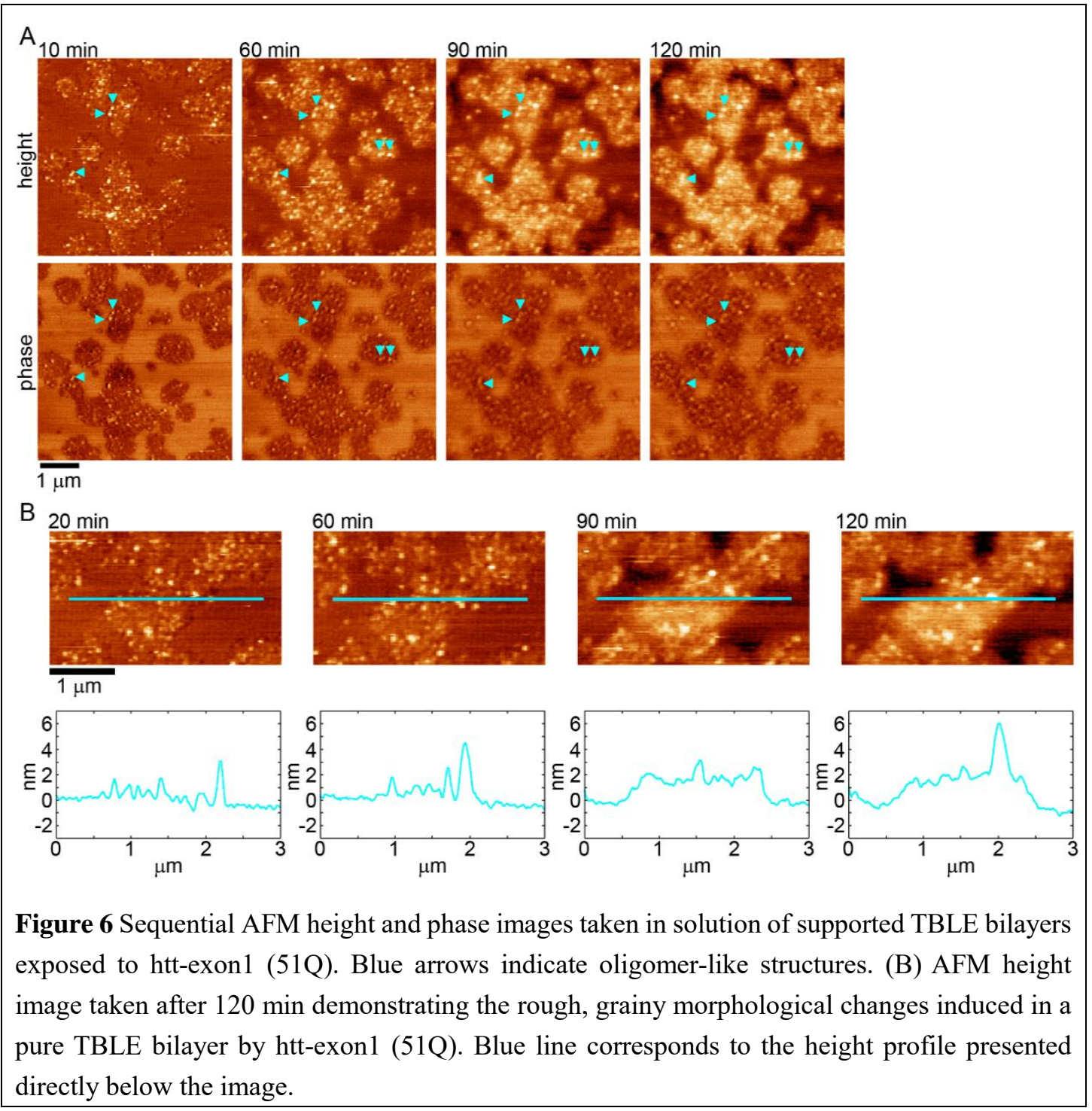

Of note, the aggregates observed on pure TBLE bilayers were globular in shape. These oligomers were fairly stable on the membrane, as they could be identified in consecutive images. Htt-exon1 (Q51) at similar concentrations aggregates into fibrils in the absence of lipid within an hour; however, there were no fibrils observed during 
the $2 \mathrm{~h}$ time course of these experiments. This suggests that the TBLE bilayers can either stabilize aggregate intermediates or promote the formation of aggregates off the regular pathway to fibrils.

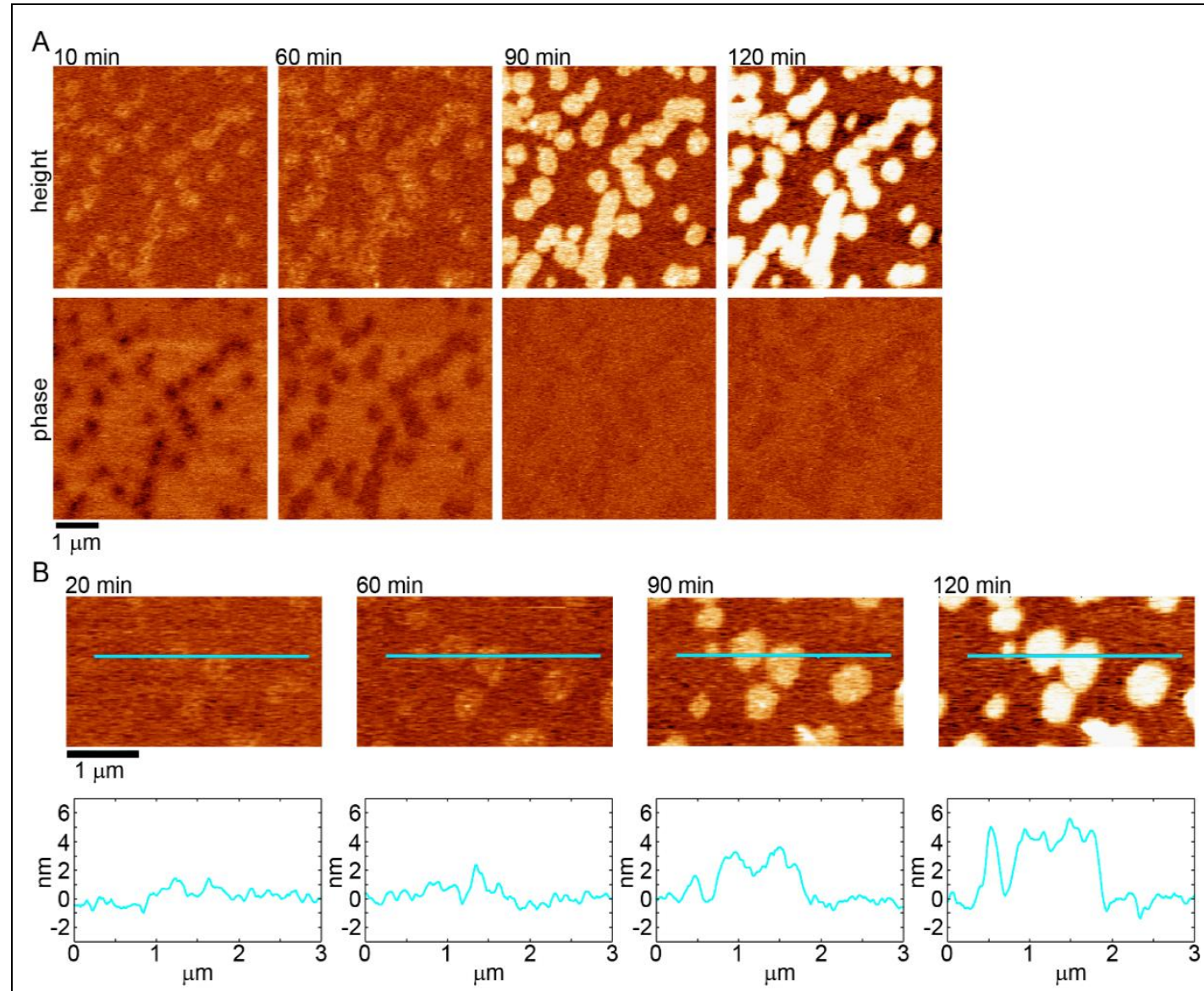

Figure 7 (A) Sequential AFM height and phase images taken in solution of supported TBLE bilayers containing $10 \%$ exogenously added cholesterol exposed to htt-exon1 (51Q). (B) AFM height image taken after 120 min demonstrating the smooth morphological changes induced in a TBLE $+10 \%$ cholesterol bilayer by htt-exon1 (51Q). Blue line corresponds to the height profile presented directly below the image.

Morphologic changes were observed on TBLE bilayers containing $10 \%$ exogenous cholesterol upon exposure to $20 \mu \mathrm{M}$ htt-exon1 (Q51) that were distinct to those occurring on pure TBLE systems. Initially, compared to the extensive disruption on pure TBLE, the disrupted regions were small and more disperse on the bilayer

(Figure 7). Slight increases in height were initially detected in these small domains 
compared to the unperturbed regions. Mechanical changes were also observed on the lipid bilayer, as indicated by phase images. With time, these domains grew in area and pronounced increase in their height were observed. Interestingly, as the domain grew, the phase contrast decreased, suggesting that the perturbed and unperturbed regions of the bilayer have similar elastic properties.

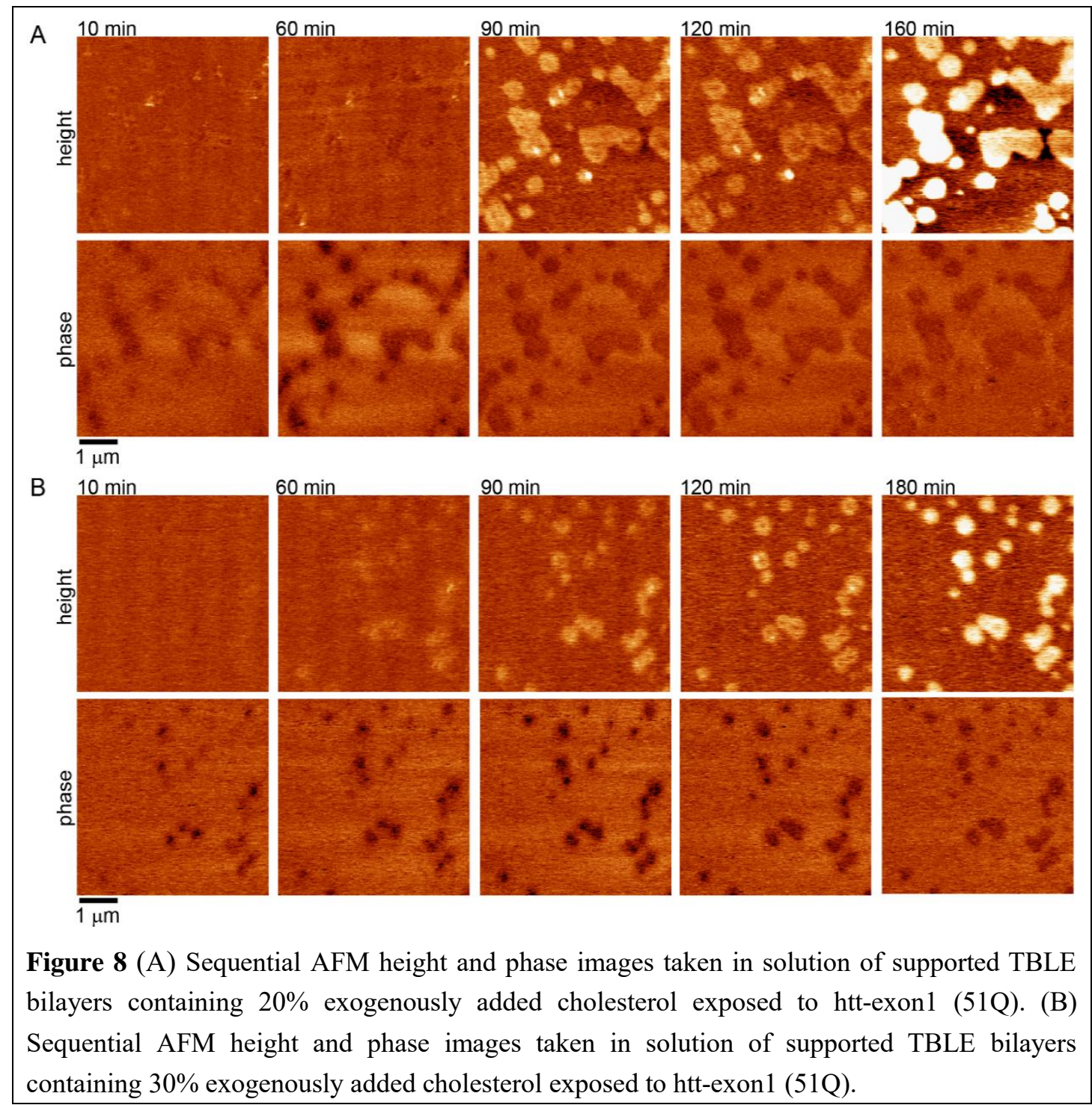

In the pure TBLE bilayer, the aggregates were grainy in appearance, but the domains in TBLE with $10 \%$ cholesterol were plateau-like with a smoother appearance.

While there were a few globular aggregates associated with these plateau-like domains, 
there was an obvious decrease in the number of such aggregates. The height of the smoother domain that developed on the TBLE containing exogenous $10 \%$ cholesterol gradually grew from $\sim 1 \mathrm{~nm}$ to $\sim 4 \mathrm{~nm}$ above the regions of unaffected regions.

Similar morphological changes in membrane topography in TBLE bilayers containing added $10 \%$ cholesterol were observed in TBLE bilayers containing $20 \%$ and $30 \%$ exogenous cholesterol

(Figure 8). Specifically, clear phase contrast was detected at initial exposure, and small domains slightly higher than the unaffected bilayer were observed. With time,

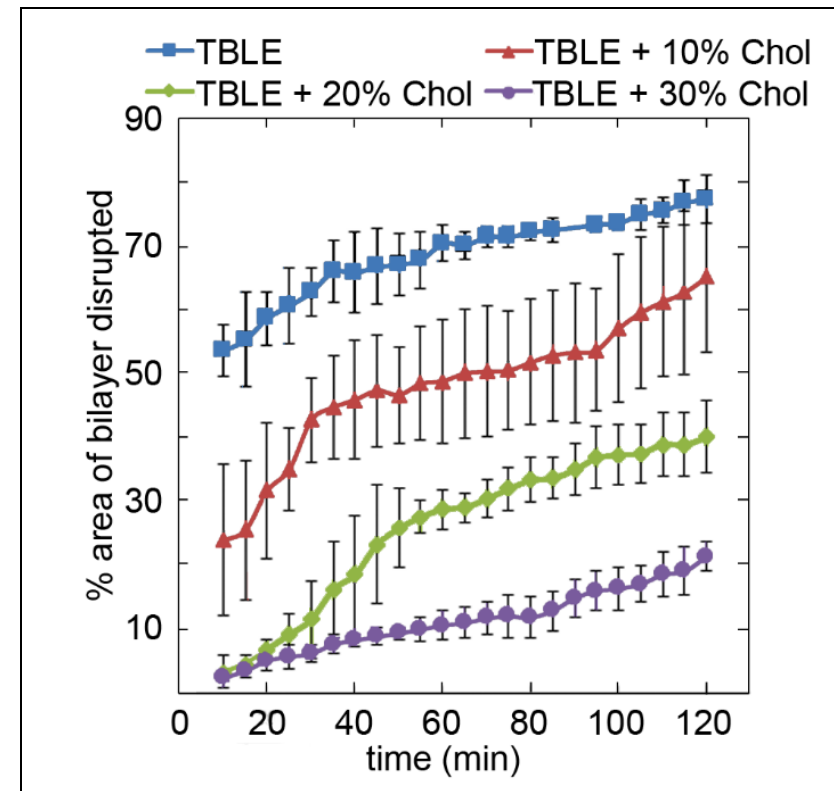

Figure 9 The percent area of disrupted bilayer morphology associated with exposure of lipid bilayers containing various amounts of cholesterol to htt-exon1(51Q) presented as a function of time. Error bars indicate one standard deviation over three separate experiments

these domains grew in size and formed a smooth plateau-like domain. The initial distinct contrast disappeared with the development of the domains in the AFM phase images. As the cholesterol content increased, surface area disrupted by exposure to httexon1 (Q51) was reduced. Also, the formation of the smooth domain was slowed. The percentage of surface with altered morphology was used to quantify the interaction of htt-exon1 (Q51) with the supported lipid bilayers. As cholesterol content increased, the extent of bilayer disruption decreased in a consistent manner with the PDA binding assay (Figure 9). 


\section{DISCUSSION}

Here, we investigated the role of cholesterol on modulating the interaction between htt and lipid membranes. Several findings have been reported. The binding of htt-exon1 (Q51) onto lipid membrane was reduce with the increase of cholesterol content in membranes. But there was still appreciable binding of htt-exon1 (Q51) to membranes even with up to $30 \%$ exogenously added cholesterol. Morphological and mechanical change also have been reported. A significant population of globular aggregates were formed when pure TBLE bilayers was exposed to htt-exon1 (Q51). The disrupted regions of the bilayer were associated with distinct contrast in AFM images, indicating altered elastic properties. Compared to grainy disrupted morphology in pure TBLE bilayers, discrete, thicker domain developed on the bilayer surface with relatively few globular htt aggregates when cholesterol content was increased. Furthermore, the extent of bilayer disruption was significantly reduced with increasing cholesterol content in lipid bilayers upon exposure to htt-exon1 (Q51).

The transcription of cholesterogenic genes is deregulated in HD. In clonal striatalderived cells that overexpress mutant htt, mRNA levels of import genes associated with cholesterol biosynthesis seem to be reduced [69]. Sterol regulatory element binding protein can promote transcription of many cholesterol biosynthesis genes, and its activity is reduced in cell and mouse models of HD. Furthermore, the activity of the synthetic enzyme HMGCoAR, which is a crucial enzyme in cholesterol biosynthesis and the level of cholesterol precursors (such as lanosterol and lathosterol), are reduced from four different HD rodent models $[52,70]$. Although the disruption of cholesterol 
homeostasis in human is generally accepted, accumulation and depletion of cholesterol are both reported [51-58]. It has been suggested that the limitation on the assays to quantify cholesterol levels may be responsible for the diverging results [71]. An increase in cholesterol content, as measured by filipin staining in YAC72 mice, was observed [72], but the application mass spectrometry techniques to assess absolute levels of cholesterol and its metabolites indicates a decrease in cholesterol content in YAC72 mice [70]. A potential decrease in cholesterol content may originate from the disruption of cholesterol biosynthesis $[52,53,56,70]$. It is suggested that the potential accumulation of cholesterol in intracellular spaces derives from the inhibition of clathrin-independent endocytosis by mutant htt [55]. Intracellular trafficking of cholesterol is impaired and cholesterol accumulates in cells. The accumulation of cholesterol in HD cellular and animal models, as in HD human brains, may contribute to NMDA-mediated excitotoxicity [57]. These finding are limited to cell and animal modes or postmortem tissues and investigations in HD patients are needed. Despite the conflicting reports on cholesterol levels in $\mathrm{HD}$, it is demonstrated that cholesterol can modulate the binding of htt to lipid membrane and aggregate formation. Also, Langmuir trough and vesicle popping experiments performed in the lab of a collaborator, Shelli L. Frey, indicate that cholesterol reduces the insertion of htt into lipid membranes and completely prevents htt-induced permeability of lipid membranes. These observations justify further studies needed to resolve the controversy about how cholesterol homeostasis is altered in HD.

Lipid rafts are special membrane domains enriched in lipid cholesterol and 
proteins. In $\mathrm{Hdh}^{\mathrm{Q} 111}$ knock in mouse model, cholesterol accumulates in membrane and membrane rafts increase when exposed to mutant htt [57]. Here, distinct domains were observed when mutant htt was exposed to bilayers with excess cholesterol that were reminiscent in some ways to lipid rafts. It is reasonable to speculate that these plateaulike domains were enriched in both htt and cholesterol. Due to the incorporation of htt protein into the domains, theses domains are much thicker than normally observed lipid raft domains in pure lipid bilayer systems [73, 74].

The initial affinity of htt to bind lipid bilayer is impeded by increasing cholesterol content in lipid membrane. The insertion of htt into lipid monolayers was also inhibited, as shown by collaborative studies with Shelli L. Frey. A condensation or a stiffening effect of adding cholesterol to the TBLE system may be a reason for these observations. Even after the htt binding to or insertion into the lipid membrane, bilayer morphology and aggregation process also are dependent on the cholesterol content.

Various aggregate species with different structures can be formed from htt. The polyQ domain in htt can itself adopt variant conformations as a monomer [75] that can lead to a variety of different aggregates [76]. Distinct monomeric conformation may play different roles in HD. For example, the monoclonal antibody 3B5H10 recognizes monomeric species of polyQ that predicts neuronal death in cell culture [77]. Various aggregates species are associated with varying levels of toxicity. Therefore, it is plausible that a subset of the aggregate conformers has a prominent role in mediating pathogenesis in HD. Since the aggregation process can be influenced by a variety of external factors, it is important to determine how the protein environment promotes the 
formation and stabilization of disease relevant htt conformations. Lipid membranes represent an important physiologically-relevant factor. The interaction of htt with membrane surfaces results in altered mechanical integrity of membranes leading to their dysfunction and stabilization or promotion of specific aggregate species $[43,65,78]$. It has been found that lipid membranes facilitate the formation of distinct aggregate species of htt fragments depending on the domains flanking the polyQ stretch [65]. Here, we demonstrate the composition of lipid membranes can modulate the aggregation of htt. Specifically, the cholesterol content in lipid bilayer promotes the formation of distinct aggregation species on lipid membranes.

Protein aggregation can disrupt membrane integrity and that is proposed as a potential mechanism associated with several neurodegenerative diseases. Induced mechanical changes in lipid membrane could underlie the membrane dysfunction observed in animal models of HD [4]. Here, it is demonstrated that the bilayer containing exogenously added cholesterol facilitates the formation of distinct domain on membrane surfaces upon exposure to htt. Interestingly, these results correlate with calcium dye leakage experiments which indicate that even a modest increase in cholesterol can dramatically alters the susceptibility of TBLE membranes to permeabilization. As the domains observed on membranes containing extra cholesterol upon exposure to htt have similar elastic properties compared to the unaltered bilayers, they may be less susceptible to leakage. These mechanical changes may result in a protective effect of additional cholesterol on the integrity of lipid membranes. Interestingly, the development of the discrete globular htt aggregates on pure TBLE 
surface exposed to htt-exon1 (Q51) correlates with increased bilayer roughness and dramatic increases in a lipid bilayer's susceptibility to permeabilization.

\section{CONCLUSION}

As a main component of lipid membrane, cholesterol plays a significant role in modulating the interaction of htt with lipid membrane. With increasing cholesterol content in lipid bilayer, the binding affinity decreases. Morphologically, distinct aggregates are formed on the lipid bilayer. Mechanically, with the addition of cholesterol in lipid bilayer, the disrupted area and unaffected area have similar mechanical properties, which may be responsible for the protective ability to reduce membrane integrity disruption.

\section{REFERENCES}

1. Chiti, F. and C.M. Dobson, Protein misfolding, functional amyloid, and human disease, in Annual Review of Biochemistry. 2006. p. 333-366.

2. Lomakin, A., et al., On the nucleation and growth of amyloid $\beta$-protein fibrils: detection of nuclei and quantitation of rate constants. Proceedings of the National Academy of Sciences USA, 1996. 93(3): p. 1125-9

3. Murphy, R.M., Peptide aggregation in neurodegenerative disease. Annual Review of Biomedical Engineering, 2002. 4: p. 155-74.

4. Burke, K.A., E.A. Yates, and J. Legleiter, Biophysical insights into how surfaces, including lipid membranes, modulate protein aggregation related to neurodegeneration. Frontiers in neurology, 2013. 4: p. 17-17.

5. Gray, J.J., The interaction of proteins with solid surfaces. Current Opinion in Structural Biology, 2004. 14(1): p. 110-115.

6. Blackley, H.K.L., et al., In-situ atomic force microscopy study of $\beta$-amyloid fibrillization. Journal of Molecular Biology, 2000. 298(5): p. 833-840. 
7. Kowalewski, T. and D.M. Holtzman, In situ atomic force microscopy study of Alzheimer's $\beta$-amyloid peptide on different substrates: New insights into mechanism of $\beta$-sheet formation. Proceedings of the National Academy of Sciences of the United States of America, 1999. 96(7): p. 3688-3693.

8. Knight, J.D. and A.D. Miranker, Phospholipid catalysis of diabetic amyloid assembly. Journal of Molecular Biology, 2004. 341(5): p. 1175-1187.

9. Yip, C.M. and J. McLaurin, Amyloid- $\beta$ peptide assembly: A critical step in fibrillogenesis and membrane disruption. Biophysical Journal, 2001. 80(3): p. 1359-1371.

10. Yip, C.M., A.A. Darabie, and J. McLaurin, A $\beta$ 42-peptide assembly on lipid Bilayers. Journal of Molecular Biology, 2002. 318(1): p. 97-107.

11. Kim, S.I., J.S. Yi, and Y.G. Ko, Amyloid $\beta$ oligomerization is induced by brain lipid rafts. Journal of Cellular Biochemistry, 2006. 99(3): p. 878-89.

12. Aisenbrey, C., et al., How is protein aggregation in amyloidogenic diseases modulated by biological membranes? European Biophysics Journal, 2008. 37(3): p. 247-55.

13. Evangelisti, E., et al., Membrane lipid composition and its physicochemical properties define cell vulnerability to aberrant protein oligomers. Journal of Cell Science, 2012. 125(Pt 10): p. 2416-27.

14. Martin, J.B. and J.F. Gusella, Huntington's disease. Pathogenesis and management. New England Journal of Medicine, 1986. 315(20): p. 1267-76.

15. Vonsattel, J.P., et al., Neuropathological classification of Huntingtonsdisease. Journal of Neuropathology and Experimental Neurology, 1985. 44(6): p. 559-577.

16. DiFiglia, M., et al., Aggregation of huntingtin in neuronal intranuclear inclusions and dystrophic neurites in brain. Science, 1997. 277(5334): p. 1990-1993.

17. Preisinger, E., et al., Evidence for a recruitment and sequestration mechanism in Huntington's disease. Philosophical Transactions of the Royal Society B: Biological Sciences, 1999. 354(1386): p. 1029-34.

18. Ravina, B., et al., The relationship between CAG repeat length and clinical progression in Huntington's disease. Movement Disorders, 2008. 23(9): p. 1223-1227. 
19. Ross, C.A. and M.A. Poirier, What is the role of protein aggregation in neurodegeneration? Nature Reviews Molecular Cell Biology, 2005. 6(11): p. 891-898.

20. Arrasate, M., et al., Inclusion body formation reduces levels of mutant huntingtin and the risk of neuronal death. Nature, 2004. 431(7010): p. 805810.

21. Wetzel, R., Physical chemistry of polyglutamine: intriguing tales of a monotonous sequence. Journal of Molecular Biology, 2012. 421(4-5): p. 466490.

22. Jayaraman, M., et al., Slow amyloid nucleation via $\alpha$-helix-rich oligomeric intermediates in short polyglutamine-containing huntingtin fragments. Journal of Molecular Biology, 2012. 415(5): p. 881-899.

23. Kar, K., et al., Critical nucleus size for disease-related polyglutamine aggregation is repeat-length dependent. Nature Structural \& Molecular Biology, 2011. 18(3): p. 328-+.

24. Landles, C., et al., Proteolysis of mutant huntingtin produces an exonl fragment that accumulates as an aggregated protein in neuronal nuclei in Huntington disease. Journal of Biological Chemimstry, 2010. 285(12): p. 8808-8823.

25. Ratovitski, T., et al., Mutant huntingtin N-terminal fragments of specific size mediate aggregation and toxicity in neuronal cells. Journal of Biological Chemistry, 2009. 284(16): p. 10855-10867.

26. Sathasivam, K., et al., Aberrant splicing of HTT generates the pathogenic exon 1 protein in Huntington disease. Proceedings of the National Academy of Sciences USA, 2013. 110(6): p. 2366-70.

27. Davies, S.W., et al., Formation of neuronal intranuclear inclusions underlies the neurological dysfunction in mice transgenic for the HD mutation. Cell, 1997. 90(3): p. 537-548.

28. Reiner, A., et al., Wild-type huntingtin plays a role in brain development and neuronal survival. Molecular Neurobiology, 2003. 28(3): p. 259-275.

29. Nasir, J., et al., Targeted disruption of the Huntington's disease gene results in embryonic lethality and behavioral and morphological changes in heterozygotes. Cell, 1995. 81(5): p. 811-23.

30. Gauthier, L.R., et al., Huntingtin controls neurotrophic support and survival of neurons by enhancing BDNF vesicular transport along microtubules. Cell, 2004. 118(1): p. 127-138. 
31. Gunawardena, S., et al., Disruption of axonal transport by loss of huntingtin or expression of pathogenic PolyQ proteins in Drosophila. Neuron, 2003. 40(1): p. 25-40.

32. Lee, W.-C.M., M. Yoshihara, and J.T. Littleton, Cytoplasmic aggregates trap polyglutamine-containing proteins and block axonal transport in a Drosophila model of Huntington's disease. Proceedings of the National Academy of Sciences of the United States of America, 2004. 101(9): p. 3224-3229.

33. Pal, A., et al., Huntingtin-HAP40 complex is a novel Rab5 effector that regulates early endosome motility and is up-regulated in Huntington's disease. Journal of Cell Biology, 2006. 172(4): p. 605-18.

34. Kegel-Gleason, K.B., Huntingtin interactions with membrane phospholipids: strategic targets for therapeutic intervention? Journal of Huntington's Disease, 2013. 2(3): p. 239-250.

35. Atwal, R.S., et al., Huntingtin has a membrane association signal that can modulate huntingtin aggregation, nuclear entry and toxicity. Human Molecular Genetics, 2007. 16(21): p. 2600-2615.

36. Hilditch-Maguire, P., et al., Huntingtin: an iron-regulated protein essential for normal nuclear and perinuclear organelles. Human Molecular Genetics, 2000. 9(19): p. 2789-2797.

37. Vidal, R., et al., Converging pathways in the occurrence of endoplasmic reticulum (ER) stress in Huntington's disease. Current Molecular Medicine, 2011. 11(1): p. 1-12.

38. Kegel, K.B., et al., Huntingtin expression stimulates endosomal-lysosomal activity, endosome tubulation, and autophagy. Journal of Neuroscience, 2000. 20(19): p. 7268-7278.

39. Qin, Z.H., et al., Huntingtin bodies sequester vesicle-associated proteins by a polyproline-dependent interaction. Journal of Neuroscience, 2004. 24(1): p. 269-281.

40. Kegel, K.B., et al., Huntingtin associates with acidic phospholipids at the plasma membrane. Journal of Biological Chemistry, 2005. 280(43): p. 3646436473.

41. Michalek, M., Evgeniy S. Salnikov, and B. Bechinger, Structure and topology of the huntingtin 1-17 membrane anchor by a combined solution and solidstate NMR approach. Biophysical Journal, 2013. 105(3): p. 699-710.

42. Kegel, K.B., et al., Polyglutamine expansion in huntingtin alters its interaction with phospholipids. Journal of Neurochemistry, 2009. 110(5): p. 1585-1597. 
43. Burke, K.A., et al., Huntingtin disrupts lipid bilayers in a polyQ-length dependent manner. Biochimica Et Biophysica Acta-Biomembranes, 2013. 1828(8): p. 1953-1961.

44. Saher, G., et al., High cholesterol level is essential for myelin membrane growth. Nature Neuroscience, 2005. 8(4): p. 468-475.

45. Ohvo-Rekila, H., et al., Cholesterol interactions with phospholipids in membranes. Progress in Lipid Research, 2002. 41(1): p. 66-97.

46. Haines, T.H., Do sterols reduce proton and sodium leaks through lipid bilayers? Progress in Lipid Research, 2001. 40(4): p. 299-324.

47. Allen, J.A., R.A. Halverson-Tamboli, and M.M. Rasenick, Lipid raft microdomains and neurotransmitter signalling. Nature Reviews Neuroscience, 2007. 8(2): p. 128-40.

48. Valenza, M. and E. Cattaneo, Emerging roles for cholesterol in Huntington's disease. Trends in Neurosciences, 2011. 34(9): p. 474-486.

49. Karasinska, J.M. and M.R. Hayden, Cholesterol metabolism in Huntington disease. Nat Rev Neurol, 2011. 7(10): p. 561-572.

50. Leoni, V. and C. Caccia, The impairment of cholesterol metabolism in Huntington disease. Biochimica et Biophysica Acta (BBA) - Molecular and Cell Biology of Lipids, 2015(0).

51. Valenza, M., et al., Dysfunction of the cholesterol biosynthetic pathway in Huntington's disease. Journal of Neuroscience, 2005. 25(43): p. 9932-9939.

52. Valenza, M., et al., Cholesterol biosynthesis pathway is disturbed in YAC128 mice and is modulated by huntingtin mutation. Human Molecular Genetics, 2007. 16(18): p. 2187-2198.

53. Valenza, M., et al., Progressive dysfunction of the cholesterol biosynthesis pathway in the R6/2 mouse model of Huntington's disease. Neurobiology of Disease, 2007. 28(1): p. 133-142.

54. Ritch, J.J., et al., Multiple phenotypes in Huntington disease mouse neural stem cells. Molecular and Cellular Neuroscience, 2012. 50(1): p. 70-81.

55. Trushina, E., et al., Mutant huntingtin inhibits clathrin-independent endocytosis and causes accumulation of cholesterol in vitro and in vivo. Human Molecular Genetics, 2006. 15(24): p. 3578-3591. 
56. Luthi-Carter, R., et al., SIRT2 inhibition achieves neuroprotection by decreasing sterol biosynthesis. Proceedings of the National Academy of Sciences of the United States of America, 2010. 107(17): p. 7927-7932.

57. del Toro, D., et al., Altered cholesterol homeostasis contributes to enhanced excitotoxicity in Huntington's disease. Journal of Neurochemistry, 2010. 115(1): p. 153-167.

58. Thakur, A.K., et al., Polyglutamine disruption of the huntingtin exon1 $N$ terminus triggers a complex aggregation mechanism. Nature Structural \& Molecular Biology, 2009. 16(4): p. 380-389.

59. Kolusheva, S., T. Shahal, and R. Jelinek, Peptide-membrane interactions studied by a new phospholipid/polydiacetylene colorimetric vesicle assay. Biochemistry, 2000. 39(51): p. 15851-9.

60. Sokolovski, M., et al., Membrane interactions and lipid binding of casein oligomers and early aggregates. Biochimical et Biophysica Acta, 2008. 1778(10): p. 2341-9.

61. Kolusheva, S., L. Boyer, and R. Jelinek, A colorimetric assay for rapid screening of antimicrobial peptides. Nature Biotechnology, 2000. 18(2): p. 225-227.

62. Zheng, F., Z. Wu, and Y. Chen, A quantitative method for the measurement of membrane affinity by polydiacetylene-based colorimetric assay. Analytica Biochemistry, 2012. 420(2): p. 171-6.

63. Yates, E.A., et al., Specific domains of $A \beta$ facilitate aggregation on and association with lipid bilayers. Journal of Molecular Biology, (0).

64. Melcher, J., et al., Origins of phase contrast in the atomic force microscope in liquids. Proceedings of the National Academy of Sciences, 2009. 106(33): p. 13655-13660.

65. Burke, K.A., et al., The interaction of polyglutamine peptides with lipid membranes is regulated by flanking sequences associated with huntingtin. Journal of Biological Chemistry, 2013.

66. Yates, E.A., et al., Specific domains of $A \beta$ facilitate aggregation on and association with lipid bilayers. Journal of Molecular Biology, 2013. 425(0): p. 1915-1933.

67. Groves, J.T., N. Ulman, and S.G. Boxer, Micropatterning fluid lipid bilayers on solid supports. Science, 1997. 275(5300): p. 651-653. 
68. Michalek, M., et al., Membrane tnteractions of the amphipathic amino terminus of huntingtin. Biochemistry, 2013.

69. Sipione, S., et al., Early transcriptional profiles in huntingtin-inducible striatal cells by microarray analyses. Human Molecular Genetics, 2002. 11(17): p. 1953-1965.

70. Valenza, M., et al., Cholesterol defect is marked across multiple rodent models of Huntington's disease and is manifest in astrocytes. Journal of Neuroscience, 2010. 30(32): p. 10844-50.

71. Marullo, M., et al., Pitfalls in the detection of cholesterol in Huntington's disease models: Technical considerations in the detection of cholesterol in Huntington's disease samples. PLOS Currents Huntington Disease, 2012. 1: p. e9a1968.

72. Trushina, E., et al., Mutant huntingtin inhibits clathrin-independent endocytosis and causes accumulation of cholesterol in vitro and in vivo. Human Molecular Genetics, 2006. 15(24): p. 3578-91.

73. Orsini, F., et al., Atomic force microscopy imaging of lipid rafts of human breast cancer cells. Biochimica et Biophysica Acta (BBA) - Biomembranes, 2012. 1818(12): p. 2943-2949.

74. Yuan, C., et al., The size of lipid rafts: an atomic force microscopy study of ganglioside GM1 domains in sphingomyelin/DOPC/cholesterol membranes. Biophysical Journal, 2002. 82(5): p. 2526-2535.

75. Kim, M.W., et al., Secondary structure of huntingtin amino-terminal region. Structure, 2009. 17(9): p. 1205-1212.

76. Nekooki-Machida, Y., et al., Distinct conformations of in vitro and in vivo amyloids of huntingtin-exon1 show different cytotoxicity. Proceedings of the National Academy of Sciences of the United States of America, 2009. 106(24): p. 9679-9684.

77. Miller, J., et al., Identifying polyglutamine protein species in situ that best predict neurodegeneration. Nature Chemical Biology, 2011. 7(12): p. 925934.

78. Burke, K.A., E.A. Yates, and J. Legleiter, Amyloid-forming proteins alter the local mechanical properties of lipid membranes. Biochemistry, 2013. 52(5): p. 808-817. 


\section{Acknowledgement}

I would like to express my deep gratitude to Dr. Justin Legleiter. I'm truly grateful of his patience, motivation, enthusiasm, and immense knowledge. Research could be tough but his encouragement and helpful instruction made it easier. His optimism would ease my anxiety. I can honestly say that he was the best advisor I could have.

Besides my advisor, I would like to thank all my colleagues. I enjoyed the discussion with Maxmore Chaibva when he or I had issues with our research. I really appreciate Albert Pilkington's help when I was stuck in China. Also I benefited a lot from working around Nicole Shamitko when I took the AFM training. I'm happy to work with Sharon Groover. It's my pleasure to work with these guys in such a perfect group.

I would like to thank the rest of my thesis committee: Prof. Blake Mertz and Prof. Stephen Valentine, for being my committee member, reading my thesis and coming to my defense.

Last but not least, I wish to thank my family who have always supported me, my parents Qitian Gao, Chunhua Wang, and most of all Yue Ren for enjoying life together with me. 